\title{
La préparation d'un article pour publication
}

\section{Georges BORDACE*}

Bien qu'il soit entendu que «ce que l'on conçoit bien » devrait « s'énoncer clairement » et que « les mots pour le dire » devraient «arriver aisément $»^{1}$, la rédaction d'un article scientifique demeure une tâche très exigeante. Afin de faciliter cette tâche, les différentes composantes d'un article ont été disséquées en 42 critères qui sont pré sentés dans la Partie I du présent travail. C es critères concernent autant le contenu que la forme d'une publication et sont présentés de façon à ce qu'ils soient applicables à différents types de recherche, qu'il sagisse d'études de type expérimental ou descriptif ou d'études d'évaluation. Après la présentation de cescritères, six étapes derédaction et de soumission d'un manuscrit pour publication sont suggérées afin de faciliter le processus d'écriture et de mise en forme de l'article (Partie II). Enfin, la liste des raisons principales invoquées par des éval uateurs externes pour recommander l'acceptation ou le refus de manuscrits soumis pour publication est présentée afin de sensibiliser les auteurs à l'égard des exigences des rédacteurs et des évaluateurs externes (Partie III).

\section{Partie I : Le contenu de l'article**}

Les composantes d'un article sont présentées selon neuf rubriques et suivent l'ordre habituel de présentation d'un article scientifique : titre, auteur(s), résumé, introduction et état de la question, matériel et méthodes, résultats, discussion et conclusion, références et considérations géné rales. La liste de ces critères peut également être utilisée comme outil de vérification (voir l'annexe en fin d'article).

\section{«Titre»}

\section{[1] Le titre reflète correctement le contenu et l'étendue de l'étude présentée}

Le titre ne doit pas induire les lecteurs en erreur. Par exemple, considérez le titre suivant : « M éthodes de définition de la note de passage pour les examens oraux $»$. En lisant I'article, le lecteur*** apprend toutefois que l'étude ne porte que sur une seule méthode d'analyse factorielle des résultats d'un seul examen en pédiatrie, administré à neuf candidats. Le sujet proposé dans le titre est donc trompeur - «note de passage » plutôt qu' « analyse factorielle »- de même que le niveau de «généralisabilité » des résultats - plusieurs méthodes (lemot «méthodes » est au pluriel dans le titre) par rapport à une seule méthode et examens oraux en général par rapport à un seul examen en pédiatrie -. Un titre plus approprié serait : «Uneana lyse factorielle des résultats d'un examen oral en pédiatrie».

\section{[2] Letitre est clair et concis et donne del'importance à l'étude}

Letitrene doit contenir que des mots clés qui captent l'attention du lecteur. La décision de lire un article reposele plus souvent sur l'attrait de son titre, qui peut être considéré comme le plus court résumé possible du travail.

\section{[3] Le titre contient à la fois une composante indicative (le quoi) et une composante informative $(\text { le message })^{3}$}

Voici un exemple d'un titre indicatif : «Une enquête sur les compétences et qualités des directeurs de programme en médecine ». Voici le même titre avec maintenant une composante informative : "Une enquête sur les compé tences et qual ités des directeurs de programmes en médecine : l'importance du leadership ». La préparation du titre peut s'effectuer en deux temps, tout d'abord en éta blissant deux listes de mots-clés, une indicative et une informative, ensuite en tentant de les combiner en créant plusieurs titres possibles. En laissant « mijoter » les titres potentiels, un titre optimal et captivant fera finalement surface.

\footnotetext{
**Traduction, avec permission de Teaching and Learning in M edicine, et miseà jour de « Considerations on Preparing a Paper for Publication $»^{2}$

***L e masculin est utilisé tout au long du texte afin d'alléger la présentation, mais sousentend égal ement le genre féminin
}

\footnotetext{
* Correspondance : Georges Bordage - Department of Medical Education - College of Medicine - University of Illinois at Chicago - 808 South Wood (MC591) - Chicago, IL 60612-7309 - Eats-Unis - Tel. 001 (312) 996-7349 - Fax 001 (312) 413-2048 -mailto:bordage@uic.edu
} 


\section{Références}

\section{«Auteur(s)»}

\section{[4] Le titre académique, les diplômes, l'affiliation et l'adresse des auteurs sont clairement indiqués}

Ces informations sont utiles pour déterminer la crédibilité des chercheurs. Bien que plusieurs revues scientifiques restreignent les informations qu'ils publient à cet égard, il est important de bien lire les instructions aux auteurs afin de fournir le maximum d'informations possibles.

\section{[5] Chaque auteur répond aux critères d'éligibilité}

Huth ${ }^{4}$ a proposé trois critères sine qua non pour établir

l'éligibilité de chaque auteur :

- avoir contribué au contenu intellectuel de l'article (soit au niveau de la planification de l'étude ou de l'interprétation des résultats) ;

- avoir participé à la rédaction de l'article ou à la révision de son contenu intellectuel ;

- être capable de défendre l'article publiquement.

(D es critères semblables ont été adoptés par le comité international des rédacteurs en chef de revues médical es5). La simple participation à la collecte des données, la supervision générale (sans apport intellectuel) ou la révision éditoriale à elles seules ne sont pas suffisantes pour être considéré comme auteur. La plupart des revues scientifiques exigent que chaque auteur ratifie la version finale du manuscrit et signe un formulaire attestant leur niveau de participation dans l'étude et la rédaction de l'article. C ertaines revues publient cette information comme partie intégrante de l'article.

\section{"Résumé»}

[6] Le résuméest complet et contient des informations précises concernant chaque élément de l'étude, c'est-àdire les buts, le contexte, le plan de recherche, les participants, l'intervention, la mesure des princi paux résultats, la présentation des résultats et les conclusions

L'utilisation d'un « résumé structuré ».6.7 est un moyen sûr d'inclure chacune des composantes de l'étude présentée. $M$ aints lecteurs prendront la décision de lire l'article en se basant sur les informations présentées dans le résumé. Ainsi, si celui-ci est incomplet, le lecteur risque de passer outre, al ors quesil avait su, par exemple, que telle méthodologie était utilisée, il aurait lu l'article en entier. (Voir Bordage \& Q uérin pour une présentation et un exemple du résumé structuré.)

\section{[ 7 ] Le résumé contient des informations précises}

$D$ es données véritables sont présentées. D es informations vagues ou générales ne sont pas utiles; par exemple, plutôt qu'écrire « $D$ egrandes différences de performance ont été trouvées. ", les auteurs auraient avantage à écrire «Les scores moyens obtenus par les étudiants lors des examens pré-clinique et clinique (150 questions à choix de réponses) furent de $87.4(s=16.2)$ et $125.7(s=11.9)$ respectivement, $p<0.01$. ».

\section{[ 8] Les conclusions et recommandations énoncées sont en accord avec l'étude menée et les résultats obtenus}

Les général isations faites à partir des résultats ne doivent pas dépasser les limites de l'étude, notamment celles induites par l'échantillonnage des participants ou par les instruments utilisés. Par exemple, il est faux d'énoncer que « Les programmes de formation à voies multiples sont meilleurs que les programmes monolithiques traditionnels » si, en réalité, il n'y a eu comparaison que de deux seuls programmes, un de chaque type.

\section{9] Des mots-clés sont présentés et représentent l'ensemble des aspects del'étude}

La liste des mots-clés doit être plus longue et englobante que les seuls mots-clés contenus dans le titre. Les motsclés renseignent sur les buts de l'étude, la méthodologie, la population visée et la nature des mesures effectuées, par exemple: « diagnostics difficiles, interprétation des données, connaissances médicales, malades simulés, internes et résidents, enseignement clinique, étude cas-témoins ».

\section{"Introduction et état de la question » [10] Le but ou les objectifs de l'étude sont clairement exprimés; la question de recherche est claire}

II est important d'établir une différence, si nécessaire, entre le but de l'étude et le but de l'article. A l'occasion, un article peut ne porter que sur certains aspects d'une étude à plus grande échelle. L'introduction contient des informations détaillées sur le problème étudié et la (les) question(s) de recherche posée(s). La question de recherche peut être énoncée soit sous forme de question, d'objectifs ou d'hypothèse de recherche, selon le type d'étude menée. La question de recherche est la pierre angulaire de toute étude et, par conséquent, doit être tout à fait claire et facile à repérer, au besoin en la formulant dans la première phrase ou le premier paragraphe. 


\section{[11] Leproblème tel qu'il est formulé, étaye par des références, est clairement en relation avec le but de l'étude}

Les auteurs doivent établir un lien clair entre ce qui est déjà connu à propos du problème abordé (l'état de la question) et la (les) question(s) de recherche proposée(s). Lechoix des références doit être judicieux et chaque réfé rence doit être justifiée, analysée ou critiquée. Une longue liste indifférenciée de références n'est pas utile ; chacune d'elles doit apporter un éclai rage particulier. Le caractère original de l'étude doit être explicite.

\section{[ 12 ] Certaines références créent un cadre conceptuel et méthodologique à l'étude}

La façon de poser la question et le choix des variables dépendent directement du cadre conceptuel (théorique) utilisé. Par exemple, les paramètres d'une étude d'évaluation seront différents selon qu'il s'agit d'une évaluation normative ou d'une évaluation critériée.

\section{[13] Les donnéess connues sont accompagnées
de références}

II est important de différencier la position des chercheurs de celle des autres auteurs et de bien attribuer les idées et découvertes à leur juste propriétaire.

\section{[ 14 ] Les variables et concepts importants sont claire ment définis}

Le lecteur doit avoir suffisamment d'informations pour bien comprendre le problème étudié et les enjeux visés. Bon nombre de lecteurs ne seront pas tout à fait familiers avec le problème ; ainsi la terminologie technique et les jargons disciplinai res doivent être rendus accessibles aux non-initiés. G ardez à l'esprit le public visé par la revue. La val eur del'étude et ses implications pratiques peuvent être sous-estimées si le lecteur ne comprend pas les termes utilisés. II est important de faire lire le manuscrit à des collègues et lecteurs potentiels qui ne sont pas familiers avec la recherche avant de soumettre le manuscrit pour publication afin d'obtenir leurs impressions et suggestions (voir la section « critique présoumission » dans la PartielI).

\section{[15] La pertinence de l'étude est argumentée}

La pertinence (importance) d'une étude peut être définie deplusieursfaçons, par exemple, par rapport aux théories et méthodes contemporaines dans le domaine ou par rapport aux bénéfices pour l'institution où la recherche a été menée.

\section{[ 16 ] Une vued'ensemble de l'étude est présentée}

Une vue d'ensemble, en fin d'introduction par exemple, permet au lecteur de sorienter et de visualiser les diffé rentes composantes de l'étude et les différentes sections de I'article. La vue d'ensemble peut être présentée de façon succincte en une ou deux phrases.

\section{«Matériel et méthodes»}

\section{[17] Les variables choisies pour l'étude sont clairement décrites et sont appropriées compte tenu de la nature de la question de recherche posée}

II doit y avoir un lien direct entre les variables choisies et la nature du problème étudié. Dans le cas d'une expérimentation, les variables indépendante (c'est-à-dire ce qui est manipulé) et dépendante (c'est-à-dire la conséquence, l'effet) sont clairement identifiées (libellées) et définies. La nature de la variable dépendante doit déterminer le type d'instrument choisi pour mesurer l'effet voulu, et non l'inverse. Trop souvent, la simple disponibilité d'un instrument détermine la nature de la variable choisie ; c'est alors «mettre la charrue devant les bœufs ». Par exemple, suite à une formation en communication médecinmalade au lit du malade, les chercheurs ont choisi de mesurer l'effet de leur intervention à l'aide de questions à choix de réponses empruntées à un examen de qualification national existant plutôt que d'utiliser, ou de développer, un instrument in vivo tel qu'une liste de vérification avec des malades simulés.

\section{[ 18 ] Le plan de recherche est décrit en détail}

Le plan de recherche comprend tous les moyens que les chercheurs ont choisis de mettre en œuvre pour répondre à la question de recherche. Bien qu'il y ait plusieurs façons de catégoriser les différents types de plan de recherche, ceux-ci peuvent être en général regroupés en pédagogie médicales selon cinq grandes catégories : 1 . les études d'observation où les chercheurs ne font qu'observer (une enquête, uneétudecas-témoins, une étude de cohortepar exemple) ; 2 . les études expérimental es où les chercheurs manipulent les variables (un essai randomisé, ou non-randomisé, avec ou sans groupe contrôle par exemple) ; 3. la recherche qualitative où les chercheurs sont des observateurs-participants qui utilisent notamment du matériel textuel issu d'entretiens individuels, de groupes de discussion ou d'observations directes en contexte naturel ; 4. les articles de synthèses (une méta-analyse, une revue historique par exemple) ; 5 . la validation d'instruments de 


\section{Références}

mesure (validité, fidélité, faisabilité par exemple). La construction du plan de recherche est, par son importance, la deuxième composante d'une étude, immédiatement après la formulation de la question de recherche.

\section{[19] Le plan de recherche est appropriéet ne contient pas de faiblesses particulières}

Le but du plan de rechercheest de contrôler le maximum de sources d'invalidité possibles, telles que des biais (c'està-dire toute erreur systématique qui favorise un groupe plutôt qu'un autre) ou desvariables confondantes (c'est-àdire toute interprétation raisonnable, autre que celles proposées par les chercheurs, qui pourrait expliquer les résultats obtenus).

Les auteurs doivent présenter la liste des sources possibles d'invalidité qu'ils ont considérées et démontrer comment ils les ont contrôlées, par des choix a priori dans leur plan de recherche ou par des analyses de contrôle a posteriori. Voici quelques exemples de sources d'invalidité: biais de sélection (par exemple, assigner des externes selon leur hôpital, al ors que certains hôpitaux pourraient favoriser leurs externes compte tenu de la nature des variables mesurées), effet de maturation des participants (par exemple, des internes et résidents qui évoluent au fur et à mesure quel'année progresse), la situation de mesure (par exemple, l'effet indice de la première mesure sur les mesures ultérieures; les participants qui changent leur comportement sachant qu'ils sont observés ou sachant ce que les chercheurs attendent d'eux), la perte de participants (par exemple, les participants ayant une piètre performance quittent l'étude, créant ainsi une fausse impression de succès) ou encore le délai entrel'intervention et la mesure de l'effet (par exemple, obtenir des résultats différents par une mesure immédiate par rapport à unemesure différée, à long terme). Ces facteurs invalidants, et tout autre facteur semblable, peuvent être contrôlés a priori avec le plan de recherche ou a posteriori par des analyses complémentaires - par exemple, en créant un groupe contrôle, en introduisant un placebo afin d'isoler la nature précise del'intervention, en effectuant des mesures multiples avant ou après l'intervention, en initiant précocement les participants à l'instrument de mesure afin d'éviter un effet possible de nouveauté, ou en créant des variables de classement et en vérifiant statistiquement si elles influencent les résultats (les hôpitaux dans le cas d'une assignation selon les hôpitaux par exemple).

\section{[20] L'instrument (les instruments) de mesure, y compris ses (leurs) qualités psychométriques, est (sont) décrit(s) clairement}

Un exemple (partiel ou complet) del'instrument de mesure est présenté, soit dans le texte del'article ou en annexe. Les qualités psychométriques des mesures obtenues comprennent leur val idité, fidélité, objectivité et précision. Ces qualités peuvent être présentées soit directement soit à l'ai de de références à des publications anté rieures. Les valeurs numériques obtenues pour les résultats de l'étude doivent être présentées.

\section{[21] La population concernée par l'étude et la méthode d'échantillonnage sont définies clairement}

La population est constituée par tous ceux à qui les chercheurs désirent généraliser leurs résultats (des médecins d'urgence certifiés et non-certifiés au Canada par exemple), alors que l'échantillon se réfère aux sujets qui ont participé à l'étude (200 médecins d'urgence recrutésà travers le C anada par exemple). Si l'échantillon est plus restreint (moins représentatif) que la population concernée (des volontaires provenant de deux provinces canadiennes par exemple), al ors les auteurs doivent présenter un argument convaincant quant à la généralisabilité de leurs résultats (validité externe). S'il y a eu une perte importante de participants, les caractéristiques des participants doivent être comparées à celles des non-participants et toutes les similitudes et différences, significatives ou non significatives, doivent être présentées. Si un échantillon de circonstance (à la portée de la main) a été utilisé, les auteurs doivent justifier leur choix et décrire leurs participants en détail afin de permettre au lecteur de juger « la généralisabilité» des résultats. La validité statistique des résultats d'une expérimentation repose sur le postulat d'une sélection aléatoire des participants à partir de la population et d'une assignation aléatoire à l'intervention. Lors d'une étude comparative, il existe deux risques : celui de conclure à tort qu'une différence existe alors qu'elle est liée au hasard et non à l'intervention (risquealpha) et celui de conclureà tort qu'une différence n'existe pas en raison d'une puissance insuffisante de l'étude (risque bêta). La prévention de ces erreurs implique que la taille idéale des échantillons soit déterminée avant l'étude sur des bases statistiques, à partir del'estimation a priori du niveau attendu de différence et de la détermination de la puissance souhaitée (c'est-à-dire 1-bêta), en général $80 \%$. 


\section{[22 ] La collecte des données est décrite clairement}

La procédure de collecte des données peut biaiser ou limiter la « généralisabilité » des résultats (l'effet de l'expérimentateur, la fidélité inter-juges, le niveau de connaissance et d'aisance avec l'instrument de mesure par exemple). L'article doit contenir suffisamment d'informations pour que le lecteur puisse reproduire l'étude.

\section{[23] Lemilieu où a eu lieu l'étude est clairement décrit}

Cette information est particulièrement utile pour le lecteur qui doit décider jusqu'à quel point les conditions de l'étude ressemblent ou non à celles de son propre milieu (le type de cursus ou d'institution hospitalo-universitaire par exemple).

\section{[ 24 ] Les méthodes d'analyse des données sont décrites de façon explicite}

La nature précise des tests statistiques doit être présentée (par exemple, remplacer «Une analyse non-paramétrique a été effectuée » par « Une anal yse de variance de Friedman à deux dimensions a été effectuée ».

\section{[ 25 ] Les méthodes d'analyse des données sont appropriées}

Le choix des tests statistiques, par exemple, dépend de la question de recherche, des hypothèses de recherche, du plan de recherche, de la nature des variables mesurées et de la robustesse du test à toute infraction aux postulats de base du test. D ans le cas de résultats «non-signficatifs » (négatifs), il est important de mentionner la puissance du test (c'est-à-dire la probabilité de détecter une différence véritable).

\section{«Résultats»}

\section{[26] D es données précises sont présentées}

Les auteurs doivent présenter des données précises, surtout concernant les résultats principaux de leur étude. D es énoncés généraux, sans données empiriques ou descriptives, sont inacceptables. Par exemple, au lieu de dire «N ous avons trouvé des différences significatives entre les deux groupes », écrire "La moyenne du groupe expérimental fut de $65 \%$ (SD $7 \%$ ) par rapport à $57 \%$ (SD $10 \%$ ) pour le groupe contrôle, $t(5,27)=2.1$, $p=0.04 »$.

\section{[ 27 ] Lestableaux et figures sont utilisés de façon judicieuse}

Les tableaux et figures sont des moyens efficaces et attirants de présenter des données complexes ou abondantes. Les données présentées dans les tableaux et figures n'ont pas à être répétées in extenso dans le texte; une fois suffit.

\section{[ 28 ] Le contenu des tableaux et figures est clair et cor- rect}

Les tableaux et figures doivent être faciles à lire et à comprendre, sans qu'il soit nécessaire de se référer au texte. Ainsi, le titre des tableaux et figures doit informer directement sur la nature des données présentées (par exemple, Tableau 2. Fréquences et pourcentages des raisons données par les étudiants.) ; les abréviations et sigles util isés doivent être explicités en annexe, immédiatement en dessous du corps des tableaux et figures. Ceux-ci ne doivent pas contenir de longues listes de données brutes, mais doivent plutôt résumer les informations (moyennes et écart-types par exemple). Les données sont correctes (par exemple, les totaux correspondent exactement aux données présentées dans le tableau ou la figure) et correspondent exactement aux données présentées dans le texte. La quantité de données présentées dans chaque tableau est raisonnable (par exemple, éviter des tableaux avec 10 colonnes et une centaine de données).

\section{[29] La section des résultats ne contient que des résul- tats; ille necontient pas d'opinion}

L'opinion des auteurs se trouve dans la discussion. Le lecteur doit pouvoir lire les résultats et se faire sa propre opinion sans l'influence des auteurs. Cette section ne contient, par ailleurs, aucun résultat issu d'une méthode qui n'aurait pas été décrite ou référencée dans la section «M atériel et méthodes ».

\section{«Discussion et Conclusion 》}

\section{[ 30 ] La discussion porte sur l'ensemble des éléments problématiques del l'étude et des résultats}

La discussion peut aller au-delà des résultats et porter sur des aspects de la méthodologie ou du cadre théorique. U ne bonne discussion peut être utile pour le lecteur voulant évaluer la pertinence du plan de recherche et la « généralisabilité » des résultats. 


\section{Références}

[ 31 ] La discussion concerne directement l'étude menée La discussion ne doit pas devenir une tribune d'opinion ne se souciant pas del'étude menée mais plutôt concerner directement certains aspects de la méthode util isée et des résultats obtenus; elle doit notamment analyser les limites de signification des résultats compte tenu des méthodes utilisées. L'attention du lecteur ne doit pas être détournée vers un autre sujet, cequi est pourtant unetentation fréquente des chercheurs lorsque les résultats ne sont pas ceux attendus. Des résultats négatifs peuvent être tout aussi importants que des résultats positifs.

\section{[ 32 ] Les données passées et présentes sont réunies dans la discussion et la conclusion}

La contribution originale des résultats de la présente étude doit être mise en exergue. Le contenu de la discussion et dela conclusion devrait répondreà la question «Et alors?».

\section{[ 33 ] Les conclusions et les recommandations propo- sées sont en accord avec l'étude menée et les résultats obtenus}

Les conclusions et recommandations ne doivent pas dépasser les limites de l'étude ; autrement dit, il ne doit pas y avoir sur-générali sation des résultats comptetenu de l'étude menée et des résultats obtenus.

\section{«Références»}

[ 34 ] Le nombre de références est raisonnable (ni trop, ni trop peul)

Une longue liste de références qui n'ont pas été analysées est inappropriée. Par contre, les lecteurs s'attendent à ce que les auteurs présentent des références qui soient pertinentes et à jour.

\section{[ 35 ] Le contenu de l'article démontre clairement que} les références ont éé lues attentivement par les auteurs Les auteurs doivent garder à l'esprit que certains lecteurs, et en particulier les évaluateurs externes, connaissent très bien la littérature, tant en ce qui concerne le contenu que l'étendue des publications. Les données connues doivent être anal ysées de façon critique et devraient conduire à une étude ayant des bases solides et une perspectiveinnovatrice.

\section{[ 36 ] Les références sont présentées selon les règles de publication}

Les règles de présentation des références peuvent varier d'une revue à une autre. Il est important de bien suivreles règles exigées dans les instructions aux auteurs.

\section{"Considérations générales》}

\section{[ 37 ] Les différentes sections del'article sont clairement identifiées et leur contenu est approprié}

Le contenu de chaque section doit correspondre exactement à son titre; par exemple, il n'y a pas dediscussion ou d'opinion dans la section des résultats ou des informations d'ordre méthodologique parmi les résultats ou dans la discussion. Lelien entre les différentes sections doit être facile à suivre. S'il y a plusieurs buts, il doit y avoir un parallélisme constant entre les différentes sections de l'article (par exemple, trois objectifs, trois résultats et trois conclusions, toujours présentés dans le même ordre).

\section{[ 38 ] Le titre des sections suit les règles exigées par la revue}

$C$ haque revue a ses propres règles de forme et de style qui sont clairement énoncées dans les instructions aux auteurs.

\section{[39] La terminologie est uniforme tout au long del'article}

Il est important de garder la même terminologie tout au long de l'article, même au risque de paraître monotone. Tout changement de terminologie peut porter à confusion ; par exemple, est-ce que « groupe » et «promotion » se réfèrent à la même entité ? Les abréviations et les unités de mesure doivent être uniformes tout au long du texte ainsi qu'entre le texte et les tableaux et figures. Évitez d'alterner entre différentes unités de mesure (heures, semaines et mois par exemple) lorsque chaque unité se réfère au même phénomène (la durée des cours par exemple).

\section{[ 40 ] Le ton de l'article dénote une approche rigoureuse dela part des auteurs}

Les lecteurs ne doivent pas avoir l'impression d'être manipulés ou orientés indûment vers un point de vue particulier. Au contraire, ils doivent avoir l'impression de recevoir des informations à partir desquelles ils pourront tirer des conclusions correctes et éclairées.

\section{[ 41] Le syle d'écritureest clair et agréable; il n'y a pas defautes d'orthographe}

Le style d'écriture n'est pas un simple embellissement mais bien l'essence même d'une expression claire et limpide. 
L'utilisation de phrases simples, ne contenant qu'une seule idée, simplifie grandement la lecture. Les paragraphes de même ne portent que sur un seul sujet, commencent par une phrase d'introduction suivie d'un corps composé de une ou plusieurs phrases et se terminent par une phrase de transition ou une conclusion. "Une idée par phrase, un sujet par paragraphe. »

Les vérificateurs informatiques orthographiques et grammaticaux sont très utiles comme outil de dépistage des erreurs (techniques) mais ne sont pas des substituts à une lecture attentive. Boileau perdure dans ce monde de haute technologie: "C'est peu qu'en un ouvrage où les fautes fourmillent des traits d'esprit semés de temps en temps pétillent $»$. Le vérificateur informatiquene peut pas dépister des mots épelés correctement mais utilisés de manière inappropriée (la frappe de «significatif » au lieu de «non significatif » ou l'emploi de mots homonymes par exemple). Ils ne détectent pas non plus si un mot manque dans une phrase. II est donc utile de faire relire le manuscrit par des collègues dont la langue maternelle est celle utilisée dans le manuscrit et ne connaissant pas directement l'étude. Ils peuvent ainsi apporter une perspective à lafoissur saformeet son contenu (voir la section «critique pré-soumission » dans la Partiell).

\section{[ 42 ] Les remerciements sont complets}

Les remerciements s'adressent le plus souvent à l'organisme subventionnaire, aux proches collaborateurs (en particulier ceux qui ne se qualifient pas comme plein auteur mais qui ont contribuéà l'étude), aux participants (qui ont donné gracieusement leur temps et leurs efforts) et aux éval uateurs externes (pour leurs précieux conseils).

\section{Partie ll : Lesétapes de rédaction et de soumission du manuscrit}

Les 42 critères discutés ci-dessus sont rarement pris en compte de façon séquentielle mais plutôt dans le cadre d'un processus itératif où les auteurs « remettent vingt fois sur le métier leur ouvrage, le polissant sans cesse et le repolissant $»$. Bien quechacun dével oppe, avec letemps et l'expérience, sa propre approche de la rédaction d'un article, certaines étapes peuvent être utiles à connaître et peuvent servir de points de repère, comme les six étapes suivantes (tirées en partie de $\mathrm{H}_{\mathrm{uth}}{ }^{8}$ ) : le démarrage, la pré rédaction, les brouillons, le peaufinage, la critique pré-soumission et la soumission du manuscrit.

\section{Le démarrage}

La mise en route d'un article est souvent vue comme une tâche redoutable, intimidante. La réponse à cinq questions peut mettre les auteurs rapidement sur la piste.

1 - En une ou deux phrases, quel est le message principal issu de l'étude?

2 - Pourquoi ce message est-il important ? Puis après ? Et alors?

3 - A qui veut-on livrer le message ? Quel public vise-ton?

4 - Q uels sont les composantes principales de l'article (résumé structuré provisoire)?

5 - Q ui sont les auteurs?

La réponse aux deux premières questions donnera un fil conducteur et un but à viser. «Q ue jamais du sujet lediscours [s'écarte] $»^{1}$. La réponse à la troisième question orientera vers des revues scientifiques (journaux) possibles. La réponse à la quatrième question sera d'abord construite en complétant de façon succincte (avec des mots-clés par exemple) chaque élément du résumé structuré. II s'agit bien d'un résumé provisoire qui donnera rapidement aux auteurs une vue d'ensemble de l'article et du travail de rédaction à venir. La réponse à la dernière question permettra d'identifier les auteurs (voir les trois critères d'éligibilité rappelés dans la rubrique «Auteurs » dans la Partiel) et de différencier entre auteurs et collaborateurs, ces derniers n'étant mentionnés que dans les remerciements. Le résumé structuré provisoire donnera aux auteurs une satisfaction immédiate et facilitera la distribution des tâches de rédaction à venir, c'est-à-dire, la détermination de qui sera responsable de quoi. L'auteur principal agira comme le maître d'œuvre. II est à noter que la position de l'auteur principal dans la liste des auteurs varie énormément d'une revue scientifique à l'autre et sel on les milieux. De façon générale, le premier auteur dans la listeest l'auteur principal, c'est-à-dire celui qui a la responsabilité générale de l'article, et souvent la responsabilité de l'étude comme telle. Enfin, les auteurs pourront se donner des échéanciers et se fixer des horaires réguliers de travail, soit de façon hebdomadaire, soit par des tranches fixes (deux jours consécutifs chaque deux semaines par exemple). Il est important de déterminer des horaires et des échéanciers qui permettront de garder le rythme de travail car malheureusement, le travail sporadique ne mène le plus souvent qu'à des recommencements perpétuels et au découragement. 


\section{Références}

\section{La prérédaction}

La prérédaction contient deux sous-étapes - lechoix des revues (à qui veut-on livrer lemessage?) et la planification détaillée du contenu de l'article (que veut-on dire aux lecteurs ?). Les travaux de recherche en pédagogie médicale sont publiés le plus souvent dans cinq types de revues scientifiques:

- les revues entièrement consacrées à la pédagogie médicale (comme la revue P édagogie M édicale) ;

- des revues générales de clinique ou de sciences de base (Revue de la M édecine G énérale par exemple);

- des revues spécialisées qui abordent notamment les aspects spécifiques de la formation des spécialistes concernés (en endocrinologie ou en chirurgie cardio-vasculaire par exemple) ou des revues locales (L eM édecin du Q uébec par exemple) ;

- des revues de santé à sujets spécifique (en épidémiologie par exemple) ;

- des revues d'éducation ou de psychologie.

Il est important d'explorer l'éventail des revues possibles et ce, pour deux raisons : il sagit d'abord de choisir la revue qui sadresse le mieux au public visé et deuxièmement de sélectionner au moins deux revues potentielles. En choisissant deux journaux et en les classant par préfé rence, les auteurs seront immédiatement prêts à soumettre leur manuscrit à nouveau au cas où ils recevraient un refus de la part de la première revue. A l'aide d'exemplaires des journaux choisis (et des informations le plus souvent disponibles sur Internet), il est important de prendre connaissance des instructions aux auteurs et de suivre le format exigé, tout en ayant à disposition deux ou trois articles modèles semblables à celui que les auteurs sapprêtent à écrire.

La deuxième composante de la phase de pré-rédaction consiste à développer un plan détaillé de l'article, section par section. La rédaction d'un article comporte trois aspects différents :

- les aspects techniques comme la ponctuation, I'orthographe, le style;

- les aspects structuraux comme l'organisation des diffé rentes sections de l'article; des paragraphes et des phrases ainsi que des liens entre les différentes composantes;

- les aspects conceptuels, c'est-à-dire les idées.

La prérédaction consiste à étayer les idées de façon détaillée, tout en utilisant les différentes sections de l'article comme points de repère. "Avant donc que d'écrire, apprenez à penser $»$.

\section{Lesbrouillons}

Arrive maintenant le temps de l'écriture proprement dite. Chaque auteur peut commencer là où il se sent le plus à I'aise. "Travaillez à loisir, quelque ordre qui vous presse». II n'est pas nécessaire de suivre l'ordre imposé par les sections de l'article. Il est souvent plus facile, par exemple, de commencer par les éléments de la méthode parce que les informations sont plus systématiques, plus techniques. Un premier brouillon est justement un brouillon. Plutôt que s'empêtrer d'emblée dans les aspects techniques et structuraux de l'écriture, il vaut mieux d'abord écrire au fil des idées. Le peaufinage viendra plus tard... : « vingt fois sur le métier... ». II est important de faire des copies des versions successives du brouillon afin de conserver certaines formulations qui sont rejetées à un moment donné, pour être ensuite reprises plus tard après mûre réflexion. "Soyez-vous à vous-même sévère critique ».

\section{Le peaufinage}

Une fois un brouillon en main, commence le raffinement du style, l'agencement des sections et l'articulation entre les phrases et les paragraphes. «Q ue le début, la fin répondent au milieu $»^{\perp}$. Vouloir se lancer trop tôt dans le raffinement risque de décourager les auteurs, voire de créer des blocages. Le peaufinageviseà donner un caractèrefini à l'article, à en faciliter la lecture et à favoriser la transmission nette et claire des messages.

\section{La critique présoumission}

Lorsque les auteurs ont un manuscrit complet en main, il est temps de le soumettre à une critique amicale en demandant à des collègues, de même langue maternelle que celle utilisée dans lemanuscrit et non familiers avec la recherche, d'en faire la lecture critique et de livrer leurs commentaires et suggestions. "Faites-vous des amis promptsà vous censurer... Aimez qu'on vous conseille, et non pas qu'on vous loue ${ }^{\prime}$. C ette première critique amicale agira comme mesure préventive et permettra d'éviter bien des contretemps ultérieurs avec le rédacteur et les éval uateurs externes. Une fois cette première critique en main, les auteurs peuvent revoir leur manuscrit et y apporter les corrections nécessaires avant de soumettre la version finale du manuscrit à la revue choisie. «Et souple à la raison, corrigez sans murmure ». 


\section{La soumission du manuscrit}

La lettre de couverture adressée au rédacteur en chef de la revue doit contenir deux messages importants : I'importance de l'étude et la justification du choix particulier de la revue. La lettre doit en somme répondreà la question : pourquoi cette revue devrait-elle publier l'article? Pour ce faire, les auteurs doivent brièvement noter pourquoi l'étude et les résultats obtenus sont importants pour le domaine spécifique et pour le public ciblé par la revue. Ils doivent aussi indiquer s'il sagit d'un sujet déjà publié dans la revue ou d'un sujet tout à fait nouveau. En mentionnant la parution antérieure de sujets semblables, les auteurs peuvent mettreen valeur la contribution originale deleur étudeainsi que les éléments de progrès et de continuité pour la revue. S'il sagit d'un sujet nouveau, les auteurs doivent convaincre le rédacteur des avantages de publier cet article pour le domaineet le public habituel de la revue.

Une fois soumis, le manuscrit subit d'abord une évaluation interne: le rédacteur en chef et les rédacteurs associés décident si le manuscrit est approprié pour la revue et, dans l'affirmative, à quels évaluateurs externes (habituellement deux ou trois, parfois quatre) le manuscrit sera envoyé pour évaluation et recommandation. Un accusé de réception est envoyé aux auteurs. Si les auteurs n'ont pas de nouvelles du rédacteur dans les trois mois suivants l'accusé de réception, il est de bon aloi de contacter le rédacteur afin de senquérir du sort du manuscrit.

Une fois les évaluations externes reçues, le rédacteur prend une décision de publier ou non l'article. (II est important de noter que les recommandations des évaluateurs externes ne sont pas simplement additionnées de façon arithmétique. Une erreur grave ou irrémédiable mentionnée par un seul éval uateur externe peut suffire pour motiver un refus). Q uatre voies sont alors possibles: - l'acceptation de l'article tel quel avec quelques corrections mineures (ce qui arrive très rarement),

- l'acceptation moyennant des corrections,

- le refus mais avec la possi bilité de le resoumettre moyennant des corrections majeures,

- ou le refus franc.

$D$ ans le cas d'une acceptation, il est important que les auteurs répondent à chacune des corrections ou suggestions exigées par le rédacteur. Souvent les corrections et suggestions sont numérotées afin de faciliter la communication. D ans le cas du refus d'un manuscrit, les auteurs peuvent profiter des commentaires des évaluateurs externes pour apporter des corrections et resoumettre le manuscrit, soit à la même revue dans le cas d'un refus conditionnel (mais pas en cas de refus franc), soit à la revue de $2^{e}$ choix identifiée lors de l'étape de la prérédaction. Enfin, il sera de la responsabilité des auteurs de vérifier que les marbres (épreuves montées) ne contiennent aucune faute. II ne restera plus alors qu'à attendre la publication et à la célébrer !

\section{Partielll : Les premiers lecteurs}

Le rédacteur en chef de la revue choisie et les évaluateurs externes seront les premiers à lire le manuscrit. D 'emblée, l'article doit leur plaire, autant par son contenu que par sa forme, et les convaincre qu'il s'agit d'un article de valeur pour la revue. Bordage a analysé les raisons les plus fré quemment citées par des évaluateurs externes au moment de proposer l'acceptation ou le rejet d'un manuscrit. Présentées de façon positive, les dix raisons principales furent (les quatre premières sont les principales) :

1 - l'étude porte sur un sujet important ;

2 - les analyses statistiques sont appropriées et optimales; 3- les résultats sont interprétés de façon complète, rien n'est passé sous silence;

4 - le texte se lit bien ;

5 - l'introduction contient un énoncé clair du problème, une revue critique et à jour de la documentation et un cadre conceptue ;

6 - les chercheurs ont utilisé un plan de recherche et des instruments de mesure optimaux ;

7 - l'étude a porté sur un échantillon adéquat et nonbiaisé;

8 - l'article contient suffisamment de données précises;

9 - les limites de l'étude sont avouées, reconnues;

10 - les implications théoriques ou méthodologiques sont présentées.

Bordage et Caelleigh (de la revue Academic M edicine) ont présidé un groupe de travail qui a proposé quelque 77 critères possibles pour l'évaluation de manuscrits de recherche $e^{10}$. C es critères sont utiles pour guider les évalua teurs externes et sensibiliser les auteurs aux exigences des rédacteurs.

Certaines revues ont rendu explicite leur politique éditoriale en publiant dansun article original les recommandations qu'elles formulaient à leurs évaluateurs externes pour l'analyse des manuscrits soumis. C'est le cas par exemple, récemment, du British M edical Journal, qui a rendu publics les critères spécifiques exigés pour les articles ayant trait à des sujets d'éducation médicale, 


\section{Références}

élaborés à leur demande par un groupe d'experts ${ }^{11}$. II est alors évidemment particulièrement judicieux pour les auteurs de manuscrits de respecter ces recommandations lorsqu'ils choisissent cette revue.

\section{En conclusion}

«Ce que l'on conçoit bien s'énonce clairement, et les mots pour le dire arrivent aisément. » La connaissance et la maîtrise des 42 critères de contenu et de forme d'un article, des six étapes de rédaction et des dix exigences principales des rédacteurs devrai ent faciliter la préparation d'un articlescientifique et par conséquent augmenter la qualité des publications dans ce domaine. La recherche est essentielle à la croissance et au développement de la pédagogie médicale, en particulier à l'élaboration de théories éducatives et d'évaluation. C'est grâce à la recherche que de nouvelles (et vieilles... ) idées peuvent être analysées et évaluées et qu'un domaine scientifique peut se protéger contre le dogmatisme et la stagnation. La publication d'un article scientifique constitue un moment culminant pour les chercheurs parce qu'elle représente l'aboutissement et la reconnaissance d'un processus long et ardu. La présentation claire des éléments de la recherche et des résultats obtenus est primordial e pour la communauté des chercheurs et pour les éducateurs sur le terrain. C en'est que par une présentation clai re, précise et transparente du plan de recherche et des analyses que d'autres chercheurs peuvent juger la qualité de l'étude et des résultats et, éventuellement explorer le sujet davantage. C'est aussi grâce à une présentation claire et non équivoque que les éducateurs peuvent apprécier à leur juste valeur les concepts et recommandations mis en avant ainsi que la pertinence des résultats pour leur propre milieu. Les résultats des travaux de recherche en pédagogie médicale pourront ainsi servir de base à la planification et à l'innovation pédagogique. Ainsi, un cycle sain et dynamique est créé entre la théorie et la pratique en pédagogie médicale: théorie - pratique - théorie... .

\section{Remerciements}

Jesuisgrandement reconnaissant au D r Jerry Colliver, rédacteur en chef dela revueTeaching and Learning in M edicine, pour la permission detraduire et de mettre à jour la Partie I del'article. Je remercie aussi vivement messi eurs les D rs Jean Jouquan (B rest) et $\mathrm{M}$ athieu $\mathrm{N}$ endaz (Genève) pour leurs précieux conseils lors de la prélecture « amicale » du manuscrit. Je suis toutefois seul responsable de la version finale. Enfin, je remerciema collègue Suzanne Poi rier, avec laquelle j'ai enseigné la rédaction scientifique au cours des années, pour sa sagesse, sa perspicacitéet sa générosité.

\section{Références}

1. Boileau N . L'Art Poétique. Paris: Classiques Larousse 1674.

2. Bordage G. Considerations on Preparing a Publication Paper. Teaching and L earning in M edicine 1989 ; $1: 47-52$.

3. H uth EJ. Types of Titles. D ans Writing and Publishing in Medicine, 3rd edition. Baltimore : Williams and Wilkins 1999 : 131-32.

4. Huth EJ. Authorship. dans Writing and Publishing in $M$ edicine, $3 r d$ edition. Baltimore: W illiams and Wilkins 1999 : 42-3.

5. International Committee of M edical Journal Editors. U niform requi rements for manuscripts submitted to biomedical journals 5th ed. JAM A $1997 ; 277$ : 927-34.

6. Huth EJ. Structured abstracts for papers reporting dinical trials. Annals of Internal M edicine 1987 ; $106: 626-27$
7. Bordage G. et $Q$ uérin $S$. Le résumé structuré: un outil de lecture, d'évaluation et de rédaction. Pédagogie M édicale $2001 ; 2: 81-4$.

8. H uth EJ Writing and Publishing in M edicine ( $3^{\text {rd }} \mathrm{ed}$.). Baltimore: Williams \& Wilkins 1999.

9. B ordage $G$. Reasons Revi ewers Rej ect and Accept $M$ anuscripts: The Stren gths and Weaknesses in M edical Education Reports. Academic M edicine 2001 ; $76: 889-96$.

10. B ordage $G$. et $C$ aelleigh $A$. Review C riteria for Research M anuscripts. Academic M edi cine 2001 ; $76: 897-78$.

11. Education Group for Guidelines on Evaluation. Guidelines for evaluating papers on educational interventions. British M edical Journal $1999 ; 318$ : 1265-67. 


\section{Liste de vérification pour préparer un article pour publication}

\section{I - Le contenu du manuscrit}

\section{Titre}

[ 1 ] Le titre reflète correctement le contenu et l'étendue de l'étude présentée.

[ 2 ] Letitre est clair et conciset donne de l'importance à l'étude.

[ 3 ] Le titre contient à la fois une composante indicative (le quoi) et une composante informative (le message).

\section{Auteurs}

[ 4 ] Le titre académique, les diplômes, l'affiliation et l'adresse des auteurs sont clai rement indiqués.

[ 5 ] Chaque auteur répond aux critères d'éligibilité.

\section{Résumés}

[ 6 ] Le résumé est complet et contient des informations précises concernant chaque élément de l'étude, c'est-à-dire les buts, le contexte, le plan de recherche, les participants, l'intervention, la mesure des principaux résultats, la présentation des résultats et les conclusions.

[ 7 ] Le résumé contient des informations précises.

[ 8 ] Les conclusions et recommandations énoncées sont en accord avec l'étude menée et les résultats obtenus.

[ 9] Des mots-clés sont présentés et représentent l'ensemble des aspects de l'étude.

\section{Introduction et état de la question}

[ 10 ] Le but ou les objectifs de l'étude sont clairement exprimés; la question de recherche est claire.

[ 11 ] Le problème tel qu'il est formulé, et étayé par des références, est clairement en relation avec lebut de l'étude.

[ 12 ] Certaines références créent un cadre conceptuel et méthodologique à l'étude.

[ 13 ] Les données connues sont accompagnées de références.

[ 14 ] Les variables et concepts importants sont clairement définis.

[ 15 ] La pertinence de l'étude est argumentée.

[ 16 ] U ne vue d'ensemble de l'étude est présentée.

\section{Matériel et méthodes}

[ 17 ] Les variables choisies pour l'étude sont clairement décrites et sont appropriées compte tenu de la nature de la question de recherche posée.

[ 18 ] Le plan de recherche est décrit en détail.

[ 19 ] Le plan de recherche est approprié et ne contient pas de faiblesses particulières.

[ 20 ] L'instrument (les instruments) de mesure, y comprisses (leurs) qualités psychométriques, est (sont) décrit(s) clairement.

[ 21 ] La population concernée par l'étude et la méthode d'échantillonnage sont définies clairement.

[ 22 ] La collecte des données est décrite clairement.

[ 23 ] Le milieu où a eu lieu l'étude est clai rement décrit.

[ 24 ] Les méthodes d'analyse des données sont décrites de façon explicite.

[ 25 ] Les méthodes d'analyse des données sont appropriées.

\section{Résultats}

[ 26 ] D es données précises sont présentées.

[ 27 ] Les tableaux et figures sont utilisés de façon judicieuse.

[ 28 ] Le contenu des tableaux et figures est clair et juste.

[ 29 ] La section des résultats ne contient que des résultats; elle ne contient pas d'opinion.

\section{Discussion et Conclusion}

[ 30 ] La discussion porte sur l'ensemble des éléments problématiques de l'étude et des résultats.

[ 31 ] La discussion concerne directement l'étude menée.

[ 32 ] L es données passées et présentes sont réunies dans la discussion et la conclusion.

[ 33 ] Les conclusions et les recommandations proposées sont en accord avec l'étude menée et les résultats obtenus.

\section{Références}

[ 34 ] Le nombre de références est raisonnable (ni trop, ni trop peu).

[ 35 ] Le contenu de l'article démontre clairement que les références ont été lues attentivement par les auteurs.

[ 36 ] Les références sont présentées selon les règles de publication. 


\section{Références}

\section{Considérations générales}

[ 37 ] Les différentes sections de l'article sont clai rement identifiées et leur contenu est approprié.

[ 38 ] Le titre des sections suit les règles exigées par la revue.

[ 39 ] La terminologie est uniforme tout au long del'article.

[ 40 ] Leton del'article dénoteuneapprocherigoureuse de la part des auteurs.

[ 41 ] Le style d'écriture est clair et agréable; ;il n'y a pas de fautes d'orthographe.

[ 42 ] Les remerciements sont complets.

\section{II - Les étapes de rédaction}

\section{Le démarrage}

- En une ou deux phrases, quel est le message principal issu del'étude?

- Pourquoi ce message est-il important ? Puis après ? Q uelle est la pertinence pour le domaine et les lecteurs?

- A qui veut-on livrer le message? Q ui est l'auditoire?

- Q uels sont les composantes principales de l'article (résumé structuré provisoire) ?

- Qui sont les auteurs?

\section{La pré-rédaction}

- Choisir au moins deux revues (à qui veut-on délivrer le message?)

- Faire un premier et deuxième choix de revue.

- Planifier de façon détaillée le contenu del'article.

\section{Les brouillons}

- Commencer là où on se sent le plus à l'aise.

- Conserver les versions successives du brouillon en les numérotant.

\section{Le peaufinage}

- Raffiner le style.

- Agencer et articuler les sections, les paragraphes, les phrases.

- D onner un caractère fini à l'article.

\section{La critique pré-soumission}

- Faire relire le manuscrit par des collègues afin d'obte nir leurs commentaires et suggestions.

- Apporter les corrections nécessaires.

\section{La soumission du manuscrit}

D ans la lettre de couverture, mentionner:

- l'importance du sujet pour le domaine,

- la pertinence du sujet pour le public de la revue et

- la continuité (ou non) du sujet dans la revue.

\section{Décision du rédacteur en chef}

\section{[ 1 ] Acceptation avec corrections mineures.}

[ 2 ] Acceptation moyennant corrections; faire toutes les corrections, révisions, une par une.

[ 3 ] Refus avec possibilité de re-soumettre; re-soumettre à la revue de $1^{\text {er }}$ ou $2^{\text {e choix. }}$

[ 4 ] Refus franc; resoumettre à la revue de $2^{\mathrm{e}}$ choix.

\section{III - R épondre aux exigences des premiers lecteurs.}

- L'étude porte sur un sujet important.

- Les analyses statistiques sont appropriées et optimales.

- Les résultats sont interprétés de façon complète; rien n'est passé sous silence.

- Le texte se lit bien.

- L'introduction contient:

- un énoncé clair du problème,

- une revue critique et à jour de la documentation et - un cadre conceptuel.

- Les chercheurs ont utilisé un plan de recherche optimal.

- Les chercheurs ont utilisé des instruments de mesure optimaux.

- L'étude a porté sur un échantillon adéquat et nonbiaisé.

- L'article contient suffisamment de données précises.

- Les limites de l'étude sont avouées, reconnues. Les implications théoriques ou méthodologiques sont présentées. 